\title{
La Geografía y la Antropología: vinculaciones en sus recorridos históricos
}

\author{
Rocío de la Canal \\ Universidad Nacional de Mar del Plata \\ @ [ rociodelacanal@gmail.com ]
}

DOI: http://dx.doi.org/10.19137/huellas-2018-2215

L a Geografía Cultural, surgida del interés de las relaciones de la humanidad con el entorno natural, se vertebra en dos ejes donde se plantean las concepciones del paisaje, sus transformaciones y arqueologías del hombre por un lado y por otro, las cuestiones centradas en los complejos procesos que definen las identidades culturales sin escindir la impronta territorial. De cierta manera y en relación con las perspectivas geográficas previas de la disciplina, la Geografía Cultural plantea un traspase del territorio a la identidad y la representación simbólica de los pueblos, las culturas y sus rasgos. Korsbaek (2007), plantea que ya Humboldt en su paso por América realizó observaciones tan rigurosas que abarcarían muchas disciplinas tales como la fitogeografía, la geografía humana, la geología, la botánica, la zoología, entre otras de carácter tanto natural como social. Por lo que el autor asume que se encuentran en estas observaciones bases sobre la imbricación entre las geografías y la Antropología. ${ }^{1}$

Algunos autores están de acuerdo en considerar que, si bien los procesos de investigación e indagación desde un carácter científico se remontan a momentos históricos tempranos, tanto la Geografía como la Antropología son nominadas en la actualidad como disciplinas modernas y jóvenes (Capel 1983, Korsbaek 2007), principalmente por su consolidación como tales ya sea en el ámbito académico como en el desarrollo científico de

1 Autores como Ortega Valcárcel (2002) o Gómez Mendoza et al. (1991), han planteado que no se puede hablar una Geografía sino de varias geografías, ya que los recorridos históricos de las diferentes orientaciones dentro de la misma disciplina han sido diferentes. Autores como Ortega, plantean que las diversas perspectivas, influencias y paradigmas son las que efectivamente dieron 'forma' a la disciplina. 
las mismas sin mencionar su vinculación interdisciplinar. Es vox populi, que la información primaria de ambas orientaciones, fue compilada por navegantes, viajeros y exploradores, exhortados por las Coronas europeas (Inglaterra, Francia, España, Portugal), deseosas de conocer las formas de habitar que existían en las tierras que colonizaban, para un dominio tanto, de los territorios como de sus agentes, con mayores conocimientos.

Los desenfrenados deseos de obtener especias y metales que se necesitaban para el desarrollo de la industria en la vieja Europa, hicieron que los reinos de España y Portugal de aquel entonces, proveyeran de los recursos económicos suficientes para emprender los viajes de exploración a las 'Indias' y al África (Halperin Donghi, 1981). En este contexto surge la necesidad de apelar a la etnografía que podía brindar herramientas para el 'acercamiento' de los habitantes de aquellos lugares y sus tradiciones (Capel, 1983).

En el caso de la Geografía se afianza entrado el siglo XIX, en las Altas Casas de Estudio de Europa con la instauración de cátedras en algunos niveles inferiores y posteriormente en los Institutos y Universidades ${ }^{2}$ y a su vez, se apoya en la conformación de Sociedades ${ }^{3}$ geográficas que aún hoy cumplen sus funciones como tales. Tanto la Geografía como la Antropología, dieron sus pasos como ciencias modernas a partir de investigadores de origen alemán en su mayoría y posteriormente británicos, que expandieron sus conocimientos a Norteamérica.

La consagración del concepto de cultura en la Geografía, sería el punto de partida que puede considerarse como tal, para rastrear las conexiones entre ambas disciplinas. Se recorren entonces dos caminos teóricos posibles: por una parte, la cultura tomada propiamente como patrón cultural, y por el otro como estructura social. Esta cuestión, llevará a afirmar que es la aplicación del concepto de cultura en las problemáticas de la ciencia geográfica, lo que efectivamente irá constituyendo el entramado de la Geografía Cultural.

La primera articulación que puede evidenciarse entre las ciencias geográficas y las ciencias antropológicas, debe remontarse a los estudios e

2 En el caso de Alemania para 1870 se contabilizaron tres cátedras de Geografía, según Capel (1981) a partir del año siguiente hasta 1890, las cátedras geográficas se fueron creando sin censar en distintas ciudades de Alemania; en Gran Bretaña la institucionalización de la Geografía fue posterior. (Para más véase Capel, 1981, cap. III y V).

3 Las Sociedades geográficas se crearon y consolidaron a lo largo del siglo XIX, la primera fue la Sociedad Geográfica de París 1821. Posteriormente en 1828 se crea la Gesellschaft für Erdkunde de Berlín en 1830. La Royal Geographical Society de Londres. Más tarde verán la luz la de México (1833), Frankfurt (1836), el instituto Brasileiro en 1838 y la Sociedad Geográfica Rusa de 1845 (Capel, 1981). 
investigaciones realizados por Friedrich Ratzel, controvertido por cientistas posteriores, debido a sus concepciones sobre el hombre emparentadas al espíritu racista de la época (Siglo XIX). Mostró un gran interés e hizo grandes aportes sobre el conocimiento de pueblos, paisajes y los modos de habitar. Si bien su formación académica estuvo más emparentada con la Historia, es en el contexto de su desarrollo como investigador que se encuentra con cuestiones que lo llevaron a interesarse por la Zoología y la Ecología y por lo tanto a enlazar lo natural con lo humano.

Sin lugar a dudas las relaciones del hombre y la naturaleza apreciadas por Ratzel, fueron encuadrando su perspectiva de análisis, hasta dar finalmente con el interesante estudio de la Geografía. Como se mencionó, la conexión entre la Antropología y la Geografía se da en sus inicios como disciplinas, teniendo lugar la instauración de cátedras universitarias, y donde el conocimiento se va generando a partir de la colección de datos provenientes de viajeros y aventureros, quienes algunos autores (Unwin 1995, Lischetti 1996) consideraron que éstos, poseían una visión sesgada de lo que veían y por tanto de la información proporcionada. Como menciona Lischetti (1996, p. 11): "el conocimiento antropológico como todos los conocimientos científicos, se constituye sin cesar a través del trabajo crítico". De ello se deriva la cuestión que plantea que "el comienzo, desarrollo y decadencia de todo sistema teórico ocurre en un ambiente que no es científicamente aséptico, sino que está permanentemente permeado por la totalidad de la vida social" (Lischetti 1996, p. 11-18).

Cabe resaltar que los estudios de Raztel se ubican en el contexto del auge de las corrientes del positivismo dominante de la época y específicamente vinculadas con las ideas evolucionistas reinantes a partir de los postulados darwinistas y spencerianos, que fomentaron las vinculaciones entre los seres vivos, la naturaleza y el homo, como menciona Claval "los grupos humanos dependen del ambiente donde están instalados: de él proviene la totalidad (para los grupos primitivos) o una gran parte de lo necesario para su subsistencia” (Claval 1999, p. 23). Las ideas raztzelianas estarán entonces, orientadas al determinismo (ambiental) geográfico, las relaciones con el entorno y la movilidad de los hombres a partir de los recursos técnicos de cada pueblo.

Como menciona Korsbaek (2007), en el primer volumen editado de la Antropogeografía (1882) se plantean de manera unilineal, las relaciones causales entre el ambiente, la cultura y la sociedad. El segundo volumen según este autor, se evidencia la impronta determinista notándose casi una reciprocidad de la naturaleza con la vida social y cultural, sin embargo, hay quienes creen que tildar de determinista a Ratzel, 'fue no comprender 
su concepción positivista' enmarcada en la época (Capel, 1983, p.286). El término de Antropogeografía acuñado por Ratzel, es según García López (2015), el que integraría una perspectiva interdisciplinaria en el ámbito de las ciencias sociales.

Los geógrafos alemanes Hahn y Richthofen, sostuvieron grandes inquietudes en relación a la cultura y el espacio. Para Ratzel el rasgo esencial que presentan los pueblos es el de la movilidad, y dicha situación estará dominada por las herramientas y técnicas que le permitan dominar la naturaleza y adaptarla (al hombre); está cuestión se verá, por lo tanto, relacionada con las ideas provenientes del darwinismo social, aunque algunos críticos posteriores pondrán esta afirmación sobre el tapete. De la misma manera, las miradas de la Geografía desde la perspectiva ratzeliana, atribuirá relevancia a los hechos culturales, articulados fuertemente con los beneficios del entorno que darán paso a los movimientos de los pueblos (Claval, 1999). Las posteriores ideas posibilistas también fueron contributivas en la vinculación en como la naturaleza modelaría las culturas humanas "considerando que marcaba los límites del desarrollo cultural" (García López, 2015, p. 164). El posibilismo geográfico específicamente el vidaliano, aportó como menciona la antropóloga García López un acercamiento a las variables de los códigos culturales en especial en lo que concierne al hombre como parte de la naturaleza y no externo a ella, lo que significa un cambio trascendente para la comprensión de los estudios sobre los modos de vida (García López, 2015, p.165).

La Geografía alemana, la cuál en sus concepciones del espacio y el paisaje, será una de las primeras maneras en acercar la Geografía y la Antropología, en los comienzos de estas investigaciones se asociarán con la Etnografía, y además abarcó el estudio de la domesticación de animales y plantas, los modos de subsistencia y la religión, poniendo especial énfasis en los hechos culturales, las herramientas y su traducción en el paisaje (Claval, 1999).

Carl Sauer, fue uno de los representantes destacados de la Geografía Cultural, entendiendo a esta disciplina como "el estudio de las prácticas de vida y de expresión que tiene el ser humano" (García López 2015, p. 166). Sauer formado en sus comienzos en instituciones de Alemania, posteriormente continuó su formación universitaria en la Universidad de Chicago y más tarde ejerció como docente investigador en la Universidad de Berkeley. De aquí podemos plantear tres trayectos históricos en el desarrollo de las ideas en la Geografía Cultural. La vinculación de Sauer con Kroeber y Lowie, ambos antropólogos, dieron lugar las inquietudes con respecto al estudio sobre los indios de los Estados Unidos y México, y sus desarrollos 
en sociedades previo al contacto hispánico. La antropóloga Luna García (1998) comenta que Sauer es el representante americano más influyente de la Geografía Cultural entre las décadas del '20 y el '60, siendo su enfoque muy cercano a la perspectiva boasiana:

Sauer y sus discípulos se interesaron por el análisis de las huellas que dejan en el paisaje natural las acciones productivas y de reproducción de diferentes grupos humanos. Para ellos el paisaje es el elemento central de estudio en geografía (Luna García, 1998, p. 72).

Diremos entonces que Sauer será el lazo durante el siglo XX que unió en una estrecha relación a la Geografía Cultural y la Antropología, y las relaciones con la Antropología norteamericana de la época, afianzadas en las ideas difusionistas de Franz Boas. Las inquietudes de Sauer se volcaron hacia un acercamiento explicativo de las implicancias de los elementos culturales principalmente los materiales, que plasmados en el territorio, otorgarían a éste un carácter específico propio. Sus investigaciones estuvieron fuertemente vinculadas a la ecología y la botánica, donde indagará acerca de las vegetaciones y sus relaciones directas con las economías de subsistencia tales como el pastoreo y la agricultura de rosa y quema. Cabe resaltar que los discípulos de Sauer, si bien tuvieron sus mismos intereses en un principio, se enfocaron a estudios de índole histórica más que contemporánea. De esta manera, los intereses de este geógrafo cultural se orientarán a las formas de apropiación del hábitat, el uso de los recursos, el impacto del hombre en la naturaleza y el origen y difusión de las culturas. El carácter difusionista, es el único aspecto de la transmisión de las culturas que se aborda en sus estudios (Claval, 1999).

Resulta interesante la postura de Sauer principalmente durante las décadas de los '30 y ' 40 donde se había orientado a la Historia y Antropología, y el giro posterior hacia la más tarde conocida como Nueva Geografía Cultural. Esta cuestión lo vinculó a las ideas epistemológicas del mundo anglosajón ya entrada la década de los 70s:

El objetivo de la escuela saueriana es por tanto la reconstrucción histórica del medio natural y de las fuerzas humanas que modifican el paisaje, la identificación de regiones culturales homogéneas definidas en base a elementos materiales (cerámica, material de construcción o tipos de viviendas) o bien elementos no materiales como religión o lenguas y dialectos; y por último el estudio de la ecología cultural histórica prestando especial atención en como la percepción y uso humano del paisaje viene condicionada por elementos culturales (Luna García, 1999, p.72). 
Las vinculaciones de la Geografía con otras ciencias sociales, nos proponen la transdisciplinariedad como metodología ineludible, los objetos de interrogación de esta ciencia, se vuelven inescindibles de los objetivos de otras ciencias sociales y también naturales. En el caso de la Antropología si tenemos en cuenta las ideas derivadas de las corrientes difusionistas, nuevamente encontramos hilos conductores. En la Geografía Cultural, tal como menciona Claval (1996), la difusión de los rasgos culturales, costumbres y tradiciones, estarán fuertemente influenciados y dependerán de la intensidad de la comunicación entre grupos.

Marvin Harris (2009) de la corriente materialista, se adentra en la cuestión del área cultural vista desde la cultura estadounidense, este concepto según el autor se originará de las prácticas en campo de la etnografía americana "que lo elaboró como un elemento heurístico para clasificar y para representar cartográficamente los grupos tribales de Norteamérica y Sudamérica (...) el concepto de área cultural es producto de la antropología norteamericana" (Harris 2009, p. 323). Este autor, considera que el concepto de cultura tiene debilidades, debido a qué si le otorga sustancial importancia al basamento geográfico natural, dejará planteado ingenuamente el determinismo geográfico, mientras que si se limita a analizar la causa de los agregados (del área cultural) se presenta como algo caprichoso. La crítica hecha por Harris entonces si bien intenta indagar las áreas culturales desde el determinismo geográfico quedan como 'cabos sueltos', ya que empíricamente se reconoce que pueblos con iguales características naturales en diferentes regiones del mundo y distantes entre sí, viven de forma muy diversa, dice: "el principal factor que priva de utilidad explicativa a una simple ordenación de culturas por áreas naturales es que lo decisivo no es simplemente el entorno, sino la interacción tecnología entorno" (Harris 2009, p. 324). Afirmamos que el concepto de Antropogeografía ratzeliano será el primer vínculo rastreado entre ambas ciencias y como menciona Luna:

La evolución de las diferentes interpretaciones de la Geografía cultural ha dado lugar a interesantes desarrollos epistemológicos en Geografía que han influido en la aparición de nuevos paradigmas, como la geografía humanística, los estudios de ecología cultural, la geografía histórica, la escuela de percepción ambiental o la geografía del comportamiento (Luna García, 1999, p.76).

Entendemos que las discusiones sobre el espacio y la emergencia de la Geografía Cultural posterior a la Segunda Guerra mundial, mostraron la carencia y por tanto necesidad tanto de cientistas sociales además de geógrafos y antropólogos, como sociólogos e historiadores, la falta de in- 
tegración y coherencia quizás en el objeto de estudio que definiera a cada una de estas disciplinas sin solaparlas y que pudieran vincular y acercar a la naturaleza y al hombre como partes inseparables, el espacio y la acción del ser humano (Korsbaek, 2007). Coincidimos también con la idea de Santos de "formación socioespacial" en donde el espacio siendo un factor social se constituye como instancia de una sociedad (Santos, 1999).

Concluimos en que en los últimos tiempos ha prevalecido la necesidad de la interdisciplinaridad más que la de delimitar el objeto de estudio, si bien el mismo definirá específicamente cada disciplina, los aportes de unas a otras enriquecen el saber científico social; coincidimos con Korsbaek en que "los estudios geográficos y antropológicos se complementan cuando se comprende la relación dialéctica que construye el hombre con su medio (Korsbaek 2007:173). Separar los campos de análisis es cada vez más dificultoso, en un universo atravesado por el carácter global del mismo, el desarrollo de las nuevas tecnologías y las dinámicas sincrónicas y diacrónicas que transita el ser humano en su historia como en su cotidianeidad.

\section{Referencias bibliográficas}

CAPEL SAEZ, H. (1983). Filosofía y Ciencia en la Geografia Contemporánea. HUROPESA, España.

CLAVAL, P. (1999). "Los fundamentos actuales de la Geografía Cultural". En Revista Documents d'analisi geográfica (34), (pp. 25-40). España.

GARCIA LOPEZ, I. C. (2015). “Apuntes para una antropología del espacio. Consideraciones desde la geografía clásica a la geografía cultural". En Revista de Antropología Experimental 15(2), (pp. 521-534), España.

GOMEZ MENDOZA, J. (1991). El pensamiento geográfico. Estudio interpretativo y antología de textos. Ed. Alianza. España.

HARRIS, M. (2009). El desarrollo de la teoría antropológica. Una historia de las teorías de la cultura. Editorial Siglo XXI. España.

HALPERIN DONGHI, T. (2013). Historia Contemporánea de América Latina. Alianza Ed. España.
KORSBAEK, L. (2007) La Antropología y el estudio de la Geografía. En Revista de Antropología. Instituto Nacional de Historia y Antropología: México.

LISCHETTI, M. (Comp. 1996). La Antropología como disciplina. 2da. Editorial EUDEBA: Argentina.

LUNA GARCÍA, A. (1999) ¿Qué hay de nuevo en la Geografía Cultural? En Revista Documents d'analisi geográfica (34) (pp. 69-80). Barcelona, España.

ORTEGA VALCÁRCEL, J. (2002). Teoría de la Geografia. Editorial Ariel: España.

SANTOS, M. (1996). La Metamorfosis del espacio habitado. Editorial Oikus Tau: España.

UNWIN, T. (1995). El lugar de la Geografía. Editorial Cátedra: España. (Créditos originales a Longman Group UK; 1992). 